\title{
Prevalence of dyslipidemia and associated risk factors among adults aged $\geq 35$ years in northern China: a cross-sectional study
}

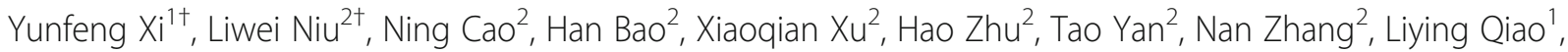
Ke Han ${ }^{1}$, Gai Hang ${ }^{1}$, Wenrui Wang ${ }^{1}$ and Xingguang Zhang ${ }^{2^{*}}$

\begin{abstract}
Background: Cardiovascular disease (CVD) prevalence has increased continuously over the last 30 years in China. Dyslipidemia is an important modifiable risk factor in CVD. We aimed to collect current data on the prevalence of dyslipidemia in northern China and explore potential influencing factors.
\end{abstract}

Methods: In this cross-sectional study, we selected a representative sample of 65,128 participants aged $\geq 35$ years in Inner Mongolia during 2015-2017. All participants completed a questionnaire and were examined for risk factors. Dyslipidemia was defined according to 2016 Chinese guidelines for adults. The associated factors for dyslipidemia were estimated by multivariate logistic regression analysis.

Results: The age-standardized prevalence of dyslipidemia was $31.2 \%$ overall, with $4.3,2.4,14.7$, and $17.4 \%$ for high total cholesterol (TC), low-density lipoprotein cholesterol (LDL-C), triglycerides (TG), and low high-density lipoprotein cholesterol (HDL-C), respectively. The dyslipidemia prevalence was significantly higher in men than women (37.9\% vs. $27.5 \%, P<0.001$ ), but postmenopausal women had a higher prevalence of dyslipidemia components (except low HDL-C). Compared with Han participants, Mongol participants had a lower prevalence of dyslipidemia (29.1\% vs. $31.4 \%, P<0.001)$. Male sex, living in urban areas, Han ethnicity, smoking, obesity, central obesity, hypertension, and diabetes were all positively correlated with dyslipidemia; alcohol consumption was linked to lower risk of dyslipidemia.

Conclusions: Our study revealed that dyslipidemia is a health problem in northern China. Greater efforts to prevent and manage dyslipidemia, especially in men under age 55 years, postmenopausal women, and people with unhealthy lifestyles or chronic diseases.

Keywords: Dyslipidemia, Prevalence, Risk factors, Northern China, Cross-sectional study

\footnotetext{
* Correspondence: zxg311@126.com

${ }^{\dagger}$ Yunfeng $\mathrm{Xi}$ and Liwei Niu contributed equally to this work.

${ }^{2}$ Public Health College, Inner Mongolia Medical University, Hohhot, Inner Mongolia Province, PR China

Full list of author information is available at the end of the article
} 


\section{Background}

With development of the economy and acceleration of urbanization, combined with aging of the population, the incidence of cardiovascular disease (CVD) has continued to increase over the last 30 years in China [1]. CVD was the top cause of death among Chinese adults in 2016, ahead of tumors and other diseases [2]. A number of studies have revealed that dyslipidemia is an important modifiable risk factor with a key role in CVD. Therefore, early screening and effective control of lipid levels can reduce the morbidity and mortality of CVD, which has useful social value $[3,4]$. The prevalence of dyslipidemia in the general population of China aged 18 and older has increased from $18.6 \%$ in 2002 to $40.4 \%$ in $2012[5,6]$. Without timely and effective control, the rate of dyslipidemia will continue to rise, leading to a heavy burden of CVD. Therefore, it is important to identify the potential influencing factors of dyslipidemia, to manage this condition and reduce the burden of CVD.

The occurrence and development of dyslipidemia frequently involves a long-term, continuous process $[7,8]$. Various factors may play different roles in the entire development process of dyslipidemia. Previous studies on dyslipidemia and associated factors have been conducted in different provinces and regions $[5,9,10]$, but large-scale investigations in northern China are scarce. The Inner Mongolia Autonomous Region is located in the north of China and includes 12 cities. Owing to its varying geographical features from east to west, the lifestyle and health problems of Inner Mongolia are representative of northern China. The prevalence and mortality of CVD are at higher levels in the region $[2,11]$. However, as an important risk factors for CVD, there are few recent comprehensive epidemiologic studies on dyslipidemia in Inner Mongolia. Our current study was designed to systematically obtain current data on the prevalence of dyslipidemia and to explore its potential influencing factors, to provide clues for the prevention and control of dyslipidemia in northern China.

\section{Methods}

\section{Study population}

Participants in our cross-sectional study were recruited from Inner Mongolia, using a convenience sampling strategy to select six sites (Hohhot, Wuhai, Chifeng, Erdos, Hulun Buir, and Xingan League) during 2015-2017. Each site was selected according to geographical, economic, and ethnic distribution factors. Participants were enrolled if they were aged $35-75$ years and lived in one of the selected regions for at least 6 of the previous 12 months. Of 70,380 enrolled participants, $5252(7.5 \%)$ were excluded because of missing data for serum lipids or body mass index (BMI). The study was approved by the ethics committee of Fuwai Hospital Chinese Academy of Medical Sciences (approval number: 2014-574). Written informed consent was obtained from each enrolled participant.

\section{Data collection and measurement}

The investigation included baseline information, physical examination, and laboratory testing. All investigators underwent professional training before the study. Information on demographic and social characteristics (such as age, sex, ethnicity, residential region, marital status, educational level, and family income), medical history (such as hypertension and diabetes), and lifestyle (such as smoking and alcohol consumption) were collected by interview where conducted at local community health service or door-to-door visits to use a standardized questionnaire.

Two consecutive blood pressure measurements were taken and the mean blood pressure value of the two readings was used. For each participant, blood pressure measurement was performed on the right upper arm after 5 min of rest, with the participant in a seated position, using an electronic sphygmomanometer (Omron, HEM-7430). Fasting blood samples were collected after at least $10 \mathrm{~h}$ of overnight fasting. Venous blood specimen was collected in Vacutainer tubes containing ethylenediaminetetraacetic acid (EDTA). Fasting plasma glucose (FPG) was analyzed enzymatically using an autoanalyzer (BeneCheck,PDG001-2). Serum total cholesterol (TC), low-density lipoprotein cholesterol (LDL-C), high-density lipoprotein cholesterol (HDL-C), triglycerides (TG) were measured by an automatic biochemical analyzer (Cardiocheck PA). All laboratory equipment was calibrated.

Height and weight were measured, with participants wearing lightweight clothing and no shoes, to the nearest $0.1 \mathrm{~cm}$ and $0.1 \mathrm{~kg}$, respectively. BMI was computed as weight $(\mathrm{kg})$ divided by the square of height $\left(\mathrm{m}^{2}\right)$. Waist circumference (WC) was measured at the level of the navel using a tape measure, to the nearest $0.1 \mathrm{~cm}$.

\section{Definitions}

According to 2016 Chinese guidelines for the management of dyslipidemia in adults [6], participants were defined as having dyslipidemia if they had one or more of the following conditions: $\mathrm{TC} \geq 6.22 \mathrm{mmol} / \mathrm{L}(240 \mathrm{mg} / \mathrm{dL})$, LDL-C $\geq$ $4.14 \mathrm{mmol} / \mathrm{L}(160 \mathrm{mg} / \mathrm{dL}), \mathrm{HDL}-\mathrm{C} \leq 1.04 \mathrm{mmol} / \mathrm{L}(40 \mathrm{mg} /$ $\mathrm{dL})$, TG $\geq 2.26 \mathrm{mmol} / \mathrm{L}(200 \mathrm{mg} / \mathrm{dL})$, or if they were taking anti-dyslipidemia medication. Hypertension was considered with systolic blood pressure (SBP) $\geq 140 \mathrm{mmHg}$ or diastolic blood pressure (DBP) $\geq 90 \mathrm{mmHg}$ or reported use of antihypertensive medication [12]. The diagnosis criterion of diabetes mellitus was FPG $\geq 7.0 \mathrm{mmol} / \mathrm{L}$ or having received treatment for diabetes [13]. Participants with BMI $\geq 28 \mathrm{~kg} /$ $\mathrm{m}^{2}$ were diagnosed with obesity, and central obesity was identified as $W C \geq 90 \mathrm{~cm}$ in men and $W C \geq 85 \mathrm{~cm}$ in women [14]. Smoking was defined as participants who smoked at least one cigarette per day during the past 12 months. Drinking was defined as drinking alcohol at least one time per month in the past 12 months. 


\section{Statistical analysis}

We described continuous variables using mean \pm standard deviation $(\mathrm{SD})$ or median and interquartile range (IQR), and the Student $t$-test or Wilcoxon rank sum test was used to compare differences. Categorical variables were shown as proportions and compared using a chi-squared test. The age-standardized prevalence was calculated according to the China 2010 census. To explore potential influencing factors of dyslipidemia, multivariate logistic regression analyses were used to calculate odds ratios (OR) and 95\% confidence intervals (CIs). All analyses were conducted using SAS version 9.3 (SAS Institute, Cary, NC, USA). Two-sided $P<0.05$ was considered statistically significant.

\section{Results}

\section{Characteristics of participants}

A total of 65,128 participants $(26,959$ men and 38,169 women) aged 35-75 years were included in our study. Characteristics of the study participants according to dyslipidemia status are shown in Table 1. Among participants, 20,
719 were diagnosed with dyslipidemia (31.8\%), with a substantial imbalance between men and women $(37.9 \%$ vs. 27.5\%). Compared with the non-dyslipidemia participants, those with dyslipidemia were more likely to be men, unmarried, and a current smoker and drinker; have older age, a high education level, higher income, and obesity; and to live in an urban area $(P<0.001)$. The mean levels of BMI, waist circumference, SBP, DBP, FPG, TC, LDL-C, and TG were significantly higher in participants with dyslipidemia than in those without dyslipidemia whereas the mean level of HDL$\mathrm{C}$ was lower in participants with dyslipidemia $(P<0.001)$.

\section{Prevalence of dyslipidemia}

Table 2 shows the prevalence of dyslipidemia among subpopulations. The prevalence of dyslipidemia was $31.8 \%$, and the prevalence of elevated TC, LDL-C, TG, and low HDL-C was 5.2, 2.9, 16.5, and $15.0 \%$, respectively. The age-standardized prevalence of dyslipidemia was $31.2 \%$; the age-standardized prevalence of elevated TC, LDL-C, TG, and decreased HDL-C was 4.3, 2.4,

Table 1 Basic characteristics of participants according to dyslipidemia status

\begin{tabular}{|c|c|c|c|c|}
\hline Variable & $\begin{array}{l}\text { Total } \\
(N=65,128)\end{array}$ & $\begin{array}{l}\text { Dyslipidemia } \\
(N=20,719)\end{array}$ & $\begin{array}{l}\text { Non-dyslipidemia } \\
(N=44,409)\end{array}$ & $P$-value \\
\hline Age (years), mean $\pm S D$ & $54.51 \pm 9.36$ & $54.78 \pm 9.20$ & $54.38 \pm 9.44$ & $<0.001$ \\
\hline Male, $n(\%)$ & $26,959(41.4)$ & $10,229(49.4)$ & $16,730(37.7)$ & $<0.001$ \\
\hline Urban, $n(\%)$ & $20,207(31.0)$ & 7517 (36.3) & $12,690(28.6)$ & $<0.001$ \\
\hline \multicolumn{5}{|l|}{ Ethnic group, $n(\%)$} \\
\hline Han & $58,628(90.0)$ & $18,753(90.5)$ & $39,875(89.8)$ & \multirow[t]{3}{*}{$<0.001$} \\
\hline Mongol & $5523(8.5)$ & $1627(7.9)$ & $3896(8.8)$ & \\
\hline Other & $977(1.5)$ & $339(1.6)$ & $638(1.4)$ & \\
\hline Married, $n(\%)$ & $59,346(91.1)$ & $18,839(90.9)$ & $40,507(91.2)$ & 0.230 \\
\hline$\geq$ High school education, $n(\%)$ & $17,960(27.6)$ & $6587(31.8)$ & $11,373(25.6)$ & $<0.001$ \\
\hline \multicolumn{5}{|l|}{ Household income(¥/year), n (\%) } \\
\hline$\leq 50,000$ & $57,713(88.6)$ & $18,024(87.0)$ & $39,689(89.4)$ & \multirow[t]{2}{*}{$<0.001$} \\
\hline$>50,000$ & $7415(11.4)$ & $2695(13.0)$ & $4720(10.6)$ & \\
\hline Smoking, $n(\%)$ & $16,060(24.7)$ & $6018(29.0)$ & $10,042(22.6)$ & $<0.001$ \\
\hline Drinking, $n(\%)$ & $12,157(18.7)$ & $4353(21.0)$ & $7804(17.6)$ & $<0.001$ \\
\hline Obesity, n (\%) & $16,300(25.0)$ & $7148(34.5)$ & 9152 (20.6) & $<0.001$ \\
\hline $\mathrm{BMI}\left(\mathrm{kg} / \mathrm{m}^{2}\right)$, mean $\pm \mathrm{SD}$ & $25.77 \pm 3.51$ & $26.88 \pm 3.30$ & $25.26 \pm 3.48$ & $<0.001$ \\
\hline Waist circumference $(\mathrm{cm})$, mean $\pm \mathrm{SD}$ & $85.62 \pm 10.05$ & $89.16 \pm 9.51$ & $84.17 \pm 9.90$ & $<0.001$ \\
\hline $\mathrm{SBP}(\mathrm{mmHg})$, mean $\pm \mathrm{SD}$ & $140.45 \pm 20.77$ & $143.04 \pm 20.48$ & $139.23 \pm 20.80$ & $<0.001$ \\
\hline $\mathrm{DBP}(\mathrm{mmHg})$, mean $\pm \mathrm{SD}$ & $84.72 \pm 11.49$ & $86.65 \pm 11.43$ & $83.82 \pm 11.41$ & $<0.001$ \\
\hline $\mathrm{FBG}(\mathrm{mmol} / \mathrm{L}), \mathrm{M}(\mathrm{IQR})$ & $6.00(5.40,6.70)$ & $6.20(5.60,7.00)$ & $5.90(5.40,6.56)$ & $<0.001$ \\
\hline $\mathrm{TC}(\mathrm{mmol} / \mathrm{L}), \mathrm{M}(\mathrm{IQR})$ & $4.48(3.89,5.14)$ & $4.61(3.86,5.61)$ & $4.44(3.90,5.01)$ & $<0.001$ \\
\hline LDL-C (mmol/L), M (IQR) & $2.40(1.90,2.96)$ & $2.48(1.92,3.24)$ & $2.37(1.90,2.86)$ & $<0.001$ \\
\hline $\mathrm{HDL}-\mathrm{C}(\mathrm{mmol} / \mathrm{L}), \mathrm{M}(\mathrm{IQR})$ & $1.37(1.13,1.66)$ & $1.03(0.92,1.36)$ & $1.47(1.26,1.74)$ & $<0.001$ \\
\hline $\mathrm{TG}(\mathrm{mmol} / \mathrm{L}), \mathrm{M}(\mathrm{IQR})$ & $1.31(0.97,1.86)$ & $2.15(1.40,2.78)$ & $1.13(0.88,1.50)$ & $<0.001$ \\
\hline
\end{tabular}

$B M I$ body mass index, SBP systolic blood pressure, $D B P$ diastolic blood pressure, $F B G$ fasting blood glucose, $T C$ total cholesterol, $L D L-C$ low-density lipoprotein cholesterol, HDL-C high-density lipoprotein cholesterol, $T G$ triglyceride, IQR interquartile range, SD standard deviation 
Table 2 Prevalence of dyslipidemia by subpopulation

\begin{tabular}{|c|c|c|c|c|c|}
\hline & Elevated TC & Elevated LDL-C & Decreased HDL-C & Elevated TG & Dyslipidemia \\
\hline Total & $5.2(5.0,5.4)$ & $2.9(2.7,3.0)$ & $16.5(16.2,16.8)$ & $15.0(14.7,15.3)$ & $31.8(31.5,32.2)$ \\
\hline Age-adjusted & $4.3(4.1,4.5)$ & $2.4(2.3,2.5)$ & $17.4(17.1,17.7)$ & $14.7(14.4,15.0)$ & $31.2(30.9,31.6)$ \\
\hline \multicolumn{6}{|l|}{ Age } \\
\hline $35-44$ & $2.0(1.8,2.3)$ & $1.2(1.0,1.4)$ & $19.9(19.2,20.7)$ & $13.9(13.3,14.6)$ & $29.8(28.9,30.7)$ \\
\hline $45-54$ & $4.6(4.3,4.9)$ & $2.5(2.3,2.7)$ & $16.9(16.4,17.4)$ & $15.5(15.1,16.0)$ & $31.3(30.7,31.9)$ \\
\hline $55-64$ & $6.7(6.4,7.1)$ & $3.7(3.4,3.9)$ & $15.5(15.0,15.9)$ & $15.6(15.2,16.1)$ & $33.5(32.9,34.1)$ \\
\hline $65-75$ & $6.7(6.2,7.1)$ & $3.6(3.2,3.9)$ & $14.4(13.7,15.1)$ & $13.5(12.9,14.2)$ & $31.5(30.6,32.4)$ \\
\hline$P$ for trend & $<0.001$ & $<0.001$ & $<0.001$ & $<0.001$ & $<0.001$ \\
\hline \multicolumn{6}{|l|}{ Gender } \\
\hline male & $2.8(2.6,3.0)$ & $1.8(1.6,2.0)$ & $26.8(26.3,27.4)$ & $15.0(14.6,15.4)$ & $37.9(37.4,38.5)$ \\
\hline female & $6.9(6.7,7.2)$ & $3.6(3.4,3.8)$ & $9.2(8.9,9.5)$ & $15.0(14.6,15.3)$ & $27.5(27.0,27.9)$ \\
\hline$P$ value & $<0.001$ & $<0.001$ & $<0.001$ & 0.906 & $<0.001$ \\
\hline \multicolumn{6}{|l|}{ Residence } \\
\hline Rural & $5.0(4.8,5.2)$ & $2.5(2.4,2.7)$ & $14.1(13.8,14.4)$ & $14.4(14.1,14.8)$ & $29.4(29.0,29.8)$ \\
\hline Urban & $5.7(5.4,6.0)$ & $3.6(3.4,3.9)$ & $21.8(21.2,22.4)$ & $16.2(157,16.7)$ & $37.2(36.5,37.9)$ \\
\hline$P$ value & $<0.001$ & $<0.001$ & $<0.001$ & $<0.001$ & $<0.001$ \\
\hline \multicolumn{6}{|l|}{ Ethnic group } \\
\hline Han & $5.2(5.0,5.3)$ & $2.8(2.7,3.0)$ & $16.6(16.3,16.9)$ & $15.1(14.8,15.4)$ & $32.0(31.6,32.4)$ \\
\hline Mongol & $5.8(5.1,6.4)$ & $3.1(2.6,3.6)$ & $15.0(14.0,15.9)^{*}$ & $13.6(12.7,14.5)^{*}$ & $29.5(28.3,30.7)^{*}$ \\
\hline Other & $5.0(3.6,6.4)$ & $3.2(2.1,4.3)$ & $20.6(18.0,23.1) *$ & $15.1(12.9,17.4)$ & $34.7(31.7,37.7)$ \# \\
\hline$P$ value & 0.163 & 0.444 & $<0.001$ & 0.008 & $<0.001$ \\
\hline \multicolumn{6}{|l|}{ Smoking } \\
\hline No & $5.8(5.6,6.0)$ & $3.1(2.9,3.3)$ & $13.7(13.4,14.0)$ & $14.8(14.4,15.1)$ & $30.0(29.6,30.4)$ \\
\hline Yes & $3.5(3.3,3.8)$ & $2.1(1.9,2.3)$ & $25.2(24.5,25.8)$ & $15.7(15.2,16.3)$ & $37.5(36.7,38.2)$ \\
\hline$P$ value & $<0.001$ & $<0.001$ & $<0.001$ & 0.003 & $<0.001$ \\
\hline \multicolumn{6}{|l|}{ Drinking } \\
\hline No & $5.4(5.3,5.6)$ & $2.9(2.8,3.1)$ & $15.4(15.1,15.7)$ & $14.4(14.1,14.7)$ & $30.9(30.5,31.3)$ \\
\hline Yes & $4.2(3.9,4.6)$ & $2.5(2.2,2.8)$ & $21.3(20.6,22.1)$ & $17.7(17.0,18.3)$ & $35.8(35.0,36.7)$ \\
\hline$P$ value & $<0.001$ & 0.005 & $<0.001$ & $<0.001$ & $<0.001$ \\
\hline \multicolumn{6}{|l|}{ Obesity } \\
\hline No & $5.0(4.8,5.2)$ & $2.8(2.6,2.9)$ & $13.9(13.6,14.2)$ & $12.5(12.2,12.8)$ & $27.8(27.4,28.2)$ \\
\hline Yes & $5.9(5.5,6.3)$ & $3.1(2.8,3.3)$ & $24.2(23.6,24.9)$ & $22.6(21.9,23.2)$ & $43.9(43.1,44.6)$ \\
\hline$P$ value & $<0.001$ & 0.051 & $<0.001$ & $<0.001$ & $<0.001$ \\
\hline \multicolumn{6}{|l|}{ Central obesity } \\
\hline No & $4.4(4.2,4.6)$ & $2.4(2.3,2.6)$ & $11.9(11.5,12.2)$ & $9.9(9.6,10.2)$ & $21.5(21.0,21.9)$ \\
\hline Yes & $6.2(5.9,6.4)$ & $3.3(3.1,3.5)$ & $21.9(21.5,22.4)$ & $21.0(20.5,21.4)$ & $38.0(37.4,38.5)$ \\
\hline$P$ value & $<0.001$ & $<0.001$ & $<0.001$ & $<0.001$ & $<0.001$ \\
\hline \multicolumn{6}{|l|}{ Hypertension } \\
\hline No & $4.0(3.7,4.2)$ & $2.3(2.1,2.5)$ & $15.3(14.9,15.8)$ & $11.1(10.8,11.5)$ & $26.2(25.7,26.7)$ \\
\hline Yes & $6.2(6.0,6.5)$ & $3.3(3.1,3.5)$ & $17.4(17.1,17.8)$ & $18.1(17.7,18.5)$ & $36.3(35.8,36.8)$ \\
\hline$P$ value & $<0.001$ & $<0.001$ & $<0.001$ & $<0.001$ & $<0.001$ \\
\hline \multicolumn{6}{|l|}{ Diabetes } \\
\hline No & $4.7(4.5,4.9)$ & $2.7(2.5,2.8)$ & $15.3(15.0,15.6)$ & $12.6(12.4,12.9)$ & $28.8(28.4,29.2)$ \\
\hline Yes & $7.3(6.8,7.7)$ & $3.7(3.4,4.0)$ & $21.7(21.0,22.4)$ & $24.8(24.1,25.6)$ & $44.5(43.6,45.4)$ \\
\hline$P$ value & $<0.001$ & $<0.001$ & $<0.001$ & $<0.001$ & $<0.001$ \\
\hline
\end{tabular}

${ }^{*}$ Compared with Han: $P<0.01 ;{ }^{\#}$ Compared with Mongol: $P<0.01$ 
14.7, and $17.4 \%$, respectively. The prevalence of dyslipidemia generally increased with age but was decreased in the age group 65-75 years. The same trend was observed for elevated LDL-C and TG. The prevalence of dyslipidemia was significantly higher in men than in women $(P<0.001)$, but we found a higher prevalence of elevated TC and LDL-C in women than in men. As shown in Fig. 1, elevated TG and dyslipidemia was more prevalent in younger men, and older women had a higher prevalence of dyslipidemia components (except decreased HDL-C), especially postmenopausal women. After age standardization, the prevalence of dyslipidemia varied among ethnic groups. In comparison with Han participants, those with Mongol ethnicity had a lower prevalence of dyslipidemia $(29.1 \%$ vs. $31.4 \%, P<0.001)$, a lower prevalence of decreased HDL-C $(16.1 \%$ vs. $17.5 \%$, $P<0.05)$, and elevated TG $(13.4 \%$ vs. $14.8 \%, P<0.05)$. Participants who smoked, drank alcohol, and had central obesity, hypertension, or diabetes had a higher prevalence of dyslipidemia and abnormal lipid levels $(P<$ $0.001)$.

\section{Influencing factors of dyslipidemia}

Figure 2 presents the results of multivariate logistic regression analysis for potential influencing factors associated with dyslipidemia. Participants who were male and those living in urban regions were more likely to have dyslipidemia and smoking was related to a higher risk of dyslipidemia. We also found that participants with obesity, central obesity, hypertension, or diabetes were more inclined to have a positively correlated with dyslipidemia. But our results showed that participants with Mongol ethnicity had a lower risk of dyslipidemia than those with Han ethnicity, and current drinkers were less likely to have dyslipidemia.
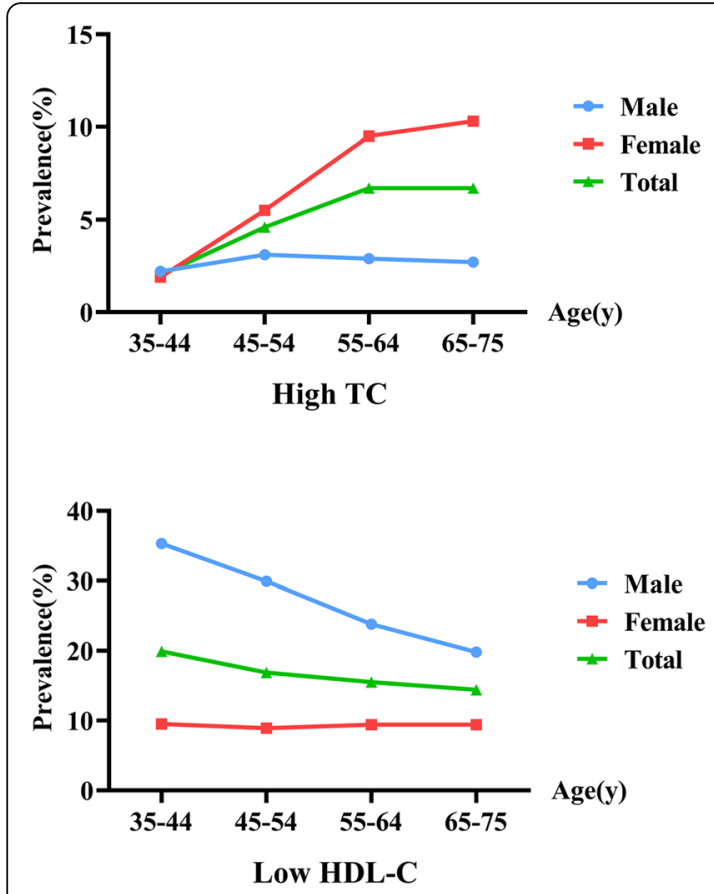
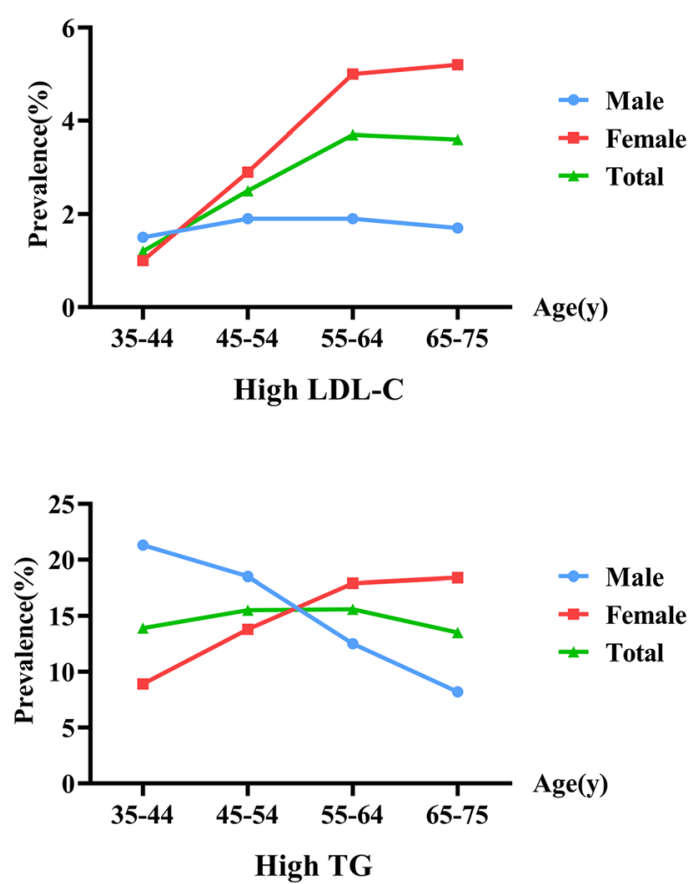

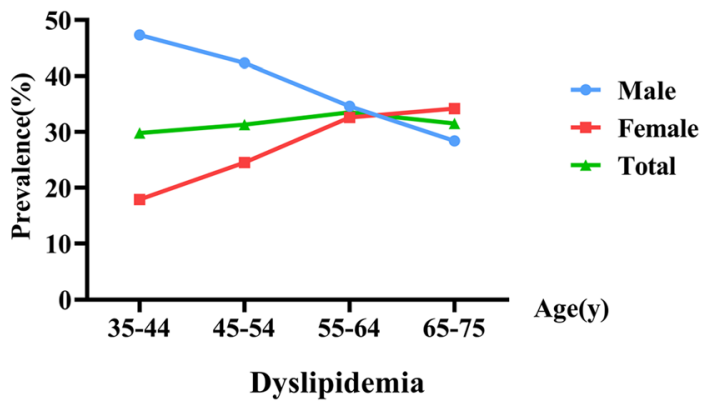

Fig. 1 Prevalence of different types of dyslipidemia by sex and age group 


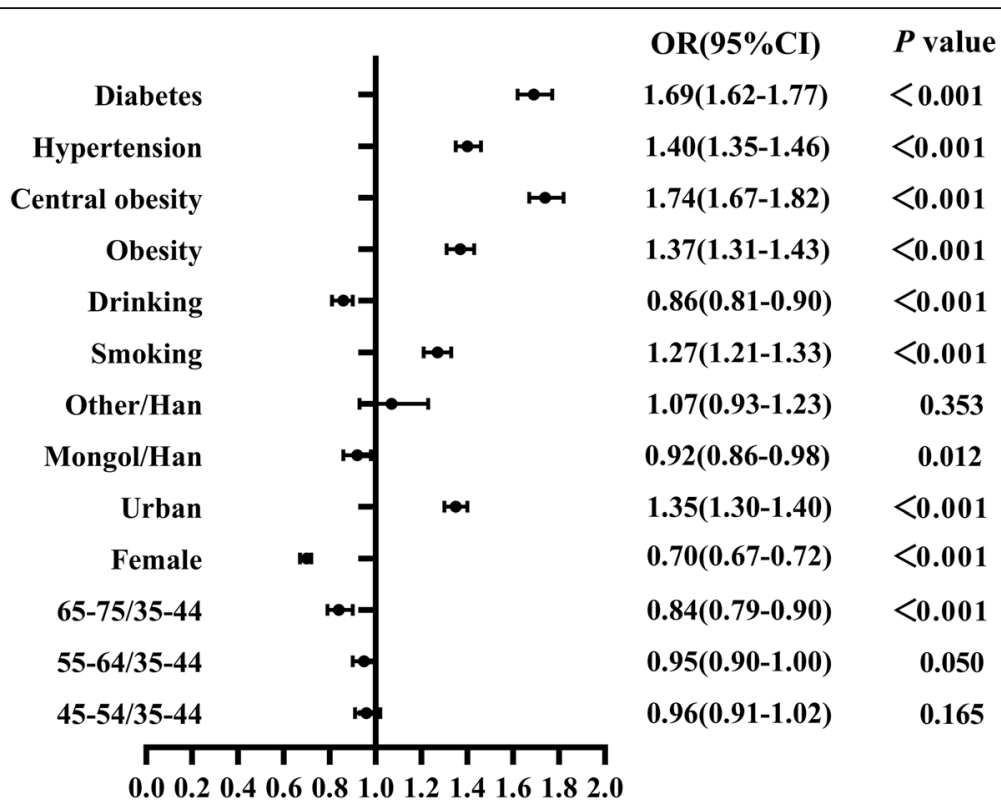

Fig. 2 Adjusted Odds Ratios (OR) of influencing factors for dyslipidemia

\section{Discussion}

A recent study showed that the annual number of deaths owing to CVD increased from 2.51 million to 3.97 million between 1990 and 2016 [2]. CVD has become the leading cause of death in China, particularly in northern China [15]. Dyslipidemia is a well-established risk factor for CVD morbidity and mortality [16]. The prevalence of dyslipidemia increased rapidly from 2002 to 2015 [1]. Identifying the epidemiological characteristics and influencing factors of dyslipidemia is an urgent public health priority. Thus, we carried out the present study of serum lipids, the largest in a representative population of Northern China.

In this study, the age-standardized prevalence of dyslipidemia was $31.2 \%$, and the prevalence rate of abnormal lipid levels was 4.3, 2.4, 14.7, and $17.4 \%$ for elevated TC, LDL-C, TG, and decreased HDL-C, respectively. A nationwide investigation showed that the prevalence of dyslipidemia was $34.7 \%$ among Chinese adults over age 35 years [17], which was higher than the results of the current study. However, compared with several regional studies [18-20], the prevalence of decreased HDL-C and elevated TG in our study were at higher levels. Located in northern China, Inner Mongolia has different food culture from other regions. Although the consumption of fresh vegetables and fruit has increased in recent years, the diet of local residents was intake meat and animal fat as the main component [21]. A recent cohort study revealed that higher fat intake was related to a greater risk of obesity [22]. Previous studies have demonstrated that the obesity could be related to low HDL$\mathrm{C}$ and elevated TG [23]. While Inner Mongolia was one of the high-prevalence cluster of obesity [24]. This could be explained that Inner Mongolia had the higher prevalence of high TG and low HDL-C. Compared to other Western countries, low HDL-C was the major subtype of dyslipidemia in China [25], and the prevalence of high TG has been shown an increasing trend for Chinese populations in recent years [1]. This was consistent with the situation that the prevalence of dyslipidemia was largely driven by HDL-C and TG in Inner Mongolia. The epidemiological data showed that HDL-C levels were negatively correlated with the risk of atherosclerotic cardiovascular disease (ASCVD) [26], and elevated TG was commonly associated with increased CVD risk [27]. Therefore, monitoring of HDL-C and TG should be strengthened to prevent the incidence of CVD among adults in northern China.

We found that prevalence of dyslipidemia increased with age but was decreased in the age group 65-75 years. Serum lipid abnormalities were more prevalent among men and participants living in urban regions, especially those under age 55-64 years. The prevalence of dyslipidemia, low HDL-C and high TG were decreased with age in men, and the different types of dyslipidemia (except decreased HDL-C) were more prevalent in postmenopausal women. The similar situations that occurred in men among different age groups have been shown in past studies $[28,29]$. Although the reason has not been confirmed, in our speculation, men may be more susceptible to risk factors when they are younger, owing to higher intake of fatty foods, greater exposure to unhealthy lifestyles, and a lack of appropriate health knowledge, resulting in men being more prone to 
dyslipidemia. Previous studies have demonstrated that the prevalence of elevated TC and LDL-C in postmenopausal women is higher than in their male counterparts [30], which is consistent with the findings of our survey. Dyslipidemia is more pronounced in older women, perhaps owing to decreased estrogen levels accompanying menopause, which may have an adverse effect on serum lipid levels in postmenopausal women [31]. As is known, dyslipidemia characterized by elevated TC or LDL-C is considered a major risk factor of ASCVD, and lowering LDL-C levels can significantly reduce the risk of morbidity and mortality owing to ASCVD $[6,32]$. To enhance the management of dyslipidemia, greater focus is needed for men under age 55 years and postmenopausal women.

We found disparities in lipid abnormalities according to ethnicity. The prevalence of decreased HDL-C, elevated TG, and dyslipidemia was higher in Han than in Mongol participants, and multivariate analysis showed that people with Mongol ethnicity had a lower risk of dyslipidemia. This finding differs from those of previous studies [33, 34]. There are different genetic backgrounds, cultures, customs, and food consumption patterns between the two ethnic groups [35]. Mongolian tend to eat more meat, milk and dairy products [34]. Some studies conducted in different countries have found that more frequent consumption of milk and dairy products could decreased the risk of dyslipidemia (elevated TG or declined HDL-C levels) [36, 37]. High consumption of milk and dairy products might related to the lower prevalence of high TG and low HDL-C levels in Mongolian.

We identified several factors that were associated with dyslipidemia in our study population. Our results that smoking, obesity, central obesity, hypertension, and diabetes can elevate the risk of dyslipidemia has been confirmed in many previous studies [29, 38]. We also found that current drinkers had a lower risk of dyslipidemia; a similar result was reported by Song [39]. Epidemiological evidence has revealed that moderate alcohol consumption can have beneficial effects on serum lipids, such as elevated HDL-C and decreased LDL-C [40], lowering the TG/HDL-C ratio [41], and that polymorphisms in alcohol-metabolizing enzymes can modify the association between alcohol intake and serum lipids [42], which may reduce the risk of CVD events [43]. However, specific levels of alcohol consumed were lacking in our study; therefore, the influence of drinking on serum lipids must be investigated further. It is recommended that people with chronic diseases or unhealthy lifestyles should be continuously monitored and managed, to effectively control dyslipidemia as much as possible.

There are several potential limitations in our study. First, a cross-sectional design rather than prospective cohort was used; thus, only associations rather than causal relationships could be determined. Second, given a lack of detailed data of dietary, alcohol consumption, physical activity, and family history of dyslipidemia, the associations of these with serum lipids could not be examined in the current study. Third, it is difficult to avoid recall bias and unmeasured confounding owing to collection of information using a questionnaire and measurement. Furthermore, a non-representative sample was used in the study. Therefore, it is not fully generalizable to all adults aged 35 years or above in northern China.

\section{Conclusions}

In summary, our study revealed that dyslipidemia is a health problem in northern China, and the dominant component of dyslipidemia was decreased HDL-C and elevated TG in people aged $\geq 35$ years. We should continuously monitor and manage lipid levels in the elderly. At the same time, the threshold of prevention should be moved forward. Special attention should be pay on the changes in lipid levels for men under age 55 years and for postmenopausal women. Dyslipidemia was more prevalent in people with Han ethnicity than those with Mongol ethnicity. Interventions for factors such as smoking, obesity, central obesity, hypertension, and diabetes can reduce the risk of dyslipidemia, and the association between drinking and serum abnormality requires further exploration. Greater efforts to prevent and manage dyslipidemia in northern China are critical to preventing the growth rate of CVD from becoming difficult to control in the future.

\section{Abbreviations}

CVD: Cardiovascular disease; TC: Total cholesterol; LDL-C: Low-density lipoprotein cholesterol; TG: Triglycerides; HDL-C: High-density lipoprotein cholesterol; BMI: Body mass index; FPG: Fasting plasma glucose; WC: Waist circumference; SBP: Systolic blood pressure; DBP: Diastolic blood pressure; ASCVD: Atherosclerotic cardiovascular disease

\section{Acknowledgments}

We express our gratefulness to all the participants and their family members for the support and cooperation, we also thank all the members and colleagues of the project team.

\section{Authors' contributions \\ YFX and LWN: developed the conception and designed the study; NC, HB and XQX: provided support in research and data collection; $H Z, T Y$ and NZ: performed data statistical analyses and interpretation; LYQ, KH and GH: contributed critically revision of the manuscript. WRW and XGZ: the guarantor of current study and take responsibility for the accuracy and integrity of data analysis. All authors have read and approved the final manuscript.}

\section{Funding}

The Ministry of Finance of China and National Health and Family Planning Commission of China; The Inner Mongolia Autonomous Region Health and Family Planning Commission Research Project [grant numbers 201701041]; The Inner Mongolia Medical University talent training and innovation team building project. The funding bodies played no role in the design of the study and collection, analysis, and interpretation of data and in writing the manuscript. 


\section{Availability of data and materials}

The raw data of the study is currently not available to publicly share because further research is underway. However, the corresponding author will consider sharing data on reasonable request.

\section{Ethics approval and consent to participate}

The study was approved by the ethics committee of Fuwai Hospital Chinese Academy of Medical Sciences (approval number: 2014-574). Written informed consent was obtained from each enrolled participant.

\section{Consent for publication}

Not applicable.

\section{Competing interests}

The authors declare that they have no competing interests.

\section{Author details}

${ }^{1}$ The Inner Mongolia Autonomous Region Comprehensive Center or Disease Control and Prevention, 50 Ordos street, Hohhot, Inner Mongolia Province 010000, P.R. China. ${ }^{2}$ Public Health College, Inner Mongolia Medical University, Hohhot, Inner Mongolia Province, PR China.

Received: 20 January 2020 Accepted: 26 June 2020

Published online: 06 July 2020

\section{References}

1. $\quad$ Song PK, Man QQ, Li H, Pang SJ, Jia SS, Li YQ, et al. Trends in lipids leve and dyslipidemia among Chinese adults, 2002-2015. Biomed Environ Sci. 2019;32(8):559-70.

2. Liu S, Li Y, Zeng X, Wang H, Yin P, Wang L, et al. Burden of cardiovascular diseases in China, 1990-2016: findings from the 2016 global burden of disease study. JAMA Cardiol. 2019:4(4):342-52

3. Lee JS, Chang PY, Zhang Y, Kizer JR, Best LG, Howard BV. Triglyceride and $\mathrm{HDL}-\mathrm{C}$ dyslipidemia and risks of coronary heart disease and ischemic stroke by glycemic dysregulation status: the strong heart study. Diabetes Care. 2017:40(4):529-37.

4. Pikula A, Beiser AS, Wang J, Himali JJ, Kelly-Hayes M, Kase CS, et al. Lipid and lipoprotein measurements and the risk of ischemic vascular events: Framingham study. Neurology. 2015;84(5):472-9.

5. He H, Yu YQ, Li Y, Kou CG, Li B, Tao YC, et al. Dyslipidemia awareness, treatment, control and influence factors among adults in the Jilin province in China: a cross-sectional study. Lipids Health Dis. 2014;13:122.

6. Joint committee for guideline revision. 2016 Chinese guidelines for the management of dyslipidemia in adults. J Geriatr Cardiol. 2018;15(1):1-29.

7. Sharma U, Kishore J, Garg A, Anand T, Chakraborty M, Lali P. Dyslipidemia and associated risk factors in a resettlement colony of Delhi. J Clin Lipidol. 2013;7(6):653-60

8. Rodriguez CJ, Daviglus ML, Swett K, González HM, Gallo LC, WassertheilSmoller S, et al. Dyslipidemia patterns among Hispanics/Latinos of diverse background in the United States. Am J Med. 2014;127(12):1186-94.

9. Shi LM, Hu JH, Zhu KL, Fu YP, Xia RM, Hu XJ. Changes of prevalence of dyslipidemia among adults: a cross-sectional study with a 2-year follow-up in urban Southeast China. Clin Lipidol. 2014;9(1):33-47.

10. Wang M, Hou X, Hu W, Chen L, Chen S. Serum lipid and lipoprotein levels of middle-aged and elderly Chinese men and women in Shandong Province. Lipids Health Dis. 2019;18(1):58.

11. Pu H, Li J, Wang P, Cui L, Wang H. The death of the circulatory system diseases in China: provincial socioeconomic and environmental perspective. Environ Sci Pollut Res Int. 2017;24(11):10381-90.

12. Joint Committee for Guideline Revision. 2018 Chinese guidelines for prevention and treatment of hypertension-a report of the revision committee of Chinese Guidelines for prevention and treatment of hypertension. J Geriatr Cardiol. 2019;16(3):182-241.

13. Chinese Diabetes Society. Guidelines for the prevention and control of type 2 diabetes in China (2017 edition). Chin J Diabetes Mellitus. 2018;10(1):4-67.

14. Bao Y, Lu J, Wang C, Yang M, Li H, Zhang X, et al. Optimal waist circumference cutoffs for abdominal obesity in Chinese. Atherosclerosis. 2008;201(2):378-84.

15. Zhou M, Wang H, Zhu J, Chen W, Wang L, Liu S, et al. Cause-specific mortality for 240 causes in China during 1990-2013: a systematic subnational analysis for the global burden of disease study 2013. Lancet. 2016:387(10015):251-72

16. Hu S, Gao R, Liu L, Zhu M, Wang W, Wang Y, et al. Summary of the 2018 report on cardiovascular diseases in China. Chinese Circ J. 2019;34(3):209-20.

17. Li S, Zhang L, Wang X, Chen Z, Dong Y, Zheng C, et al. Status of dyslipidemia among adults aged 35 years and above in China. Chinese Circ J. 2019;34(7):681-7.

18. Zhang J, Tang J, Guo X, Dong J, Ren J, Chen X, et al. Prevalence of dyslipidemia among residents aged 18-69 years in Shandong province of China,2011. Zhonghua Yu Fang Yi Xue Za Zhi. 2016;50(3):230-4.

19. Yuan X, Ni W, Wang R, Chi H, Sun Y, Lv D, et al. 6-Year trends in lipids among adults in Shenzhen, China. J Public Health (Oxf). 2019;fdz113.

20. Zhang FL, Xing YQ, Wu YH, Liu HY, Luo Y, Sun MS, et al. The prevalence, awareness, treatment, andcontrol of dyslipidemia in Northeast China: a population-based cross-sectional survey. Lipids Health Dis. 2017;16(1):61.

21. Wang X, Liu A, Maolin D, Wu J, Wang W, Qian Y, et al. Diet quality is associated with reduced risk of hypertension among Inner Mongolia adults in northern China. Public Health Nutr. 2020;23(9):1543-54.

22. Cao YJ, Wang HJ, Zhang B, Qi SF, Mi YJ, Pan XB, et al. Associations of fat and carbohydrate intake with becoming overweight and obese: an 11-year longitudinal cohort study. Br J Nutr. 2020:1-14.

23. Htet AS, Kjøllesdal MK, Aung WP, Moe Myint AN, Aye WT, Wai MM, et al. Lipid profiles and determinants of total cholesterol and hypercholesterolaemia among 25-74 year-old urban and rural citizens of the Yangon Region, Myanmar: a cross-sectional study. BMJ Open. 2017;7(11): e017465.

24. Zhang X, Zhang M, Zhao Z, Huang Z, Deng Q, Li Y, et al. Geographic variation in prevalence of adult obesity in China: results from the 2013-2014 National Chronic Disease and risk factor surveillance. Ann Intern Med. 2020; 172(4):291-3.

25. Lu Y, Wang P, Zhou T, Lu J, Spatz ES, Nasir K, et al. Comparison of Prevalence, Awareness, Treatment, and Control of Cardiovascular Risk Factors in China and the United States. J Am Heart Assoc. 2018;7(3): e007462.

26. Feiq JE, Hewing B, Smith JD, Hazen SL, Fisher EA. High-density lipoprotein and atherosclerosis regression: evidence from preclinical and clinical studies. Circ Res. 2014;114(1):205-13.

27. Toth PP, Fazio S, Wong ND, Hull M, Nichols GA. Risk of cardiovascular events in patients with hypertriglyceridemia: a review of real-world evidence. Diabetes Obes Metab. 2020;22(3):279-89.

28. Sun GZ, Li Z, Guo L, Zhou Y, Yang HM, Sun YX. High prevalence of dyslipidemia and associated risk factors among rural Chinese adults. Lipids Health Dis. 2014;13:189.

29. Liu X, Yu S, Mao Z, Li Y, Zhang H, Yang K, et al. Dyslipidemia prevalence, awareness, treatment, control, and risk factors in Chinese rural population: the Henan rural cohort study. Lipids Health Dis. 2018;17(1):119.

30. Pan L, Yang Z, Wu Y, Yin RX, Liao Y, Wang J, et al. The prevalence, awareness, treatment and control of dyslipidemia among adults in China. Atherosclerosis. 2016:248:2-9.

31. Ambikairajah A, Walsh E, Cherbuin N. Lipid profile differences during menopause: a review with meta-analysis. Menopause. 2019;26(11):1327-33.

32. Katzmann JL, Laufs U. New insights in the control of low-density lipoprotein cholesterol to prevent cardiovascular disease. Curr Cardiol Rep. 2019;21(8): 69.

33. Li G, Guo G, Wang W, Wang K, Wang H, Dong F, et al. Association of prehypertension and cardiovascular risk factor clustering in Inner Mongolia: a cross-sectional study. BMJ Open. 2017;7(6):e015340.

34. Zhou L, Zhao X, Heizhati M, Abulikemu S, Zhang D, Cheng Q, et al. Trends in lipids and lipoproteins among adults in northwestern Xinjiang, China, from 1998 through 2015. J Epidemiol. 2019;29(7):257-63.

35. Li G, Wang H, Wang K, Wang W, Dong F, Qian Y, et al. Prevalence, awareness, treatment, control and risk factors related to hypertension among urban adults in Inner Mongolia 2014: differences between Mongolian and Han populations. BMC Public Health. 2016;16:294.

36. Merino J, Mateo-Gallego R, Plana N, Bea AM, Ascaso J, Lahoz C, et al. Lowfat dairy products consumption is associated with lower triglyceride concentrations in a Spanish hypertriglyceridemic cohort. Nutr Hosp. 2013; 28(3):927-33.

37. Song S, Paik HY, Park M, Song Y. Dyslipidemia patterns are differentially associated with dietary factors. Clin Nutr. 2016:35(4):885-91. 
38. Lin $H Q$, Wu JY, Chen ML, Chen FQ, Liao YJ, Wu YT, et al. Prevalence of dyslipidemia and prediction of 10-year CVD risk among older adults living in southeast coastal regions in China: a cross-sectional study. Clin Interv Aging. 2019;14:1119-29.

39. Song $P$, Zha $M$, Yang $X, X u Y$, Wang $H$, Fang Z, et al. Socioeconomic and geographic variations in the prevalence, awareness, treatment and control of dyslipidemia in middle-aged and older Chinese. Atherosclerosis. 2019; 282:57-66.

40. Brinton EA. Effects of ethanol intake on lipoproteins. Curr Atheroscler Rep. 2012;14(2):108-14.

41. Wakabayashi I. Alcohol intake and triglycerides/high-density lipoprotein cholesterol ratio in men with hypertension. Am J Hypertens. 2013;26(7):88895.

42. Sasakabe T, Wakai K, Kawai S, Hishida A, Naito M, Suzuki S, et al. Modification of the associations of alcohol intake with serum low-density lipoprotein cholesterol and triglycerides by ALDH2 and ADH1B polymorphisms in Japanese men. J Epidemiol. 2018;28(4):185-93.

43. Ronksley PE, Brien SE, Turner BJ, Mukamal KJ, Ghali WA. Association of alcohol consumption with selected cardiovascular disease outcomes: a systematic review and meta-analysis. BMJ. 2011;342:d671.

\section{Publisher's Note}

Springer Nature remains neutral with regard to jurisdictional claims in published maps and institutional affiliations.

Ready to submit your research? Choose BMC and benefit from:

- fast, convenient online submission

- thorough peer review by experienced researchers in your field

- rapid publication on acceptance

- support for research data, including large and complex data types

- gold Open Access which fosters wider collaboration and increased citations

- maximum visibility for your research: over $100 \mathrm{M}$ website views per year

At BMC, research is always in progress.

Learn more biomedcentral.com/submissions 